\title{
A numerical analysis of a steady equation of a power-law fluid flow on a moving wall.
}

\author{
B. A. Peter and R. O. Ayeni \\ Department of Pure and Applied Mathematics, Lautech,Ogbomoso.
}

\begin{abstract}
We present a paper on the numerical analysis of a power-law fluid flow on a moving wall. We investigate the steady boundary-layer flow of a non-Newtonian fluid, represented by a power-law model over a moving flat plate. The governing partial differential equation is transformed into ordinary differential equation through a similarity variable. The finite difference method is employed to obtain solution of the non-linear problem. We investigate the effect of the power-law viscosity index $n$, and the results obtained are discussed.

Keywords: Steady boundary-layer flow; Moving plate; Non - Newtonian power-law fluid and Finite difference method.
\end{abstract}

\section{Introduction}

The forced convection flow over a moving fluid has many practical engineering applications such as the cooling of polymer films or sheets and metallic plates on conveyers. Accurate and comprehensive computational techniques such as finite difference method can be applied to solve partial differential equations that model the flow of a power-law fluid.

There have been several studied on the power-law fluid flow on a moving flat plate. Abdelhafez[1] studied skin friction and heat transfer on a continuous flat surface moving in a parallel free stream. Abel et al. [2] investigated the effects of thermal buoyancy and variable thermal conductivity on the MHD flow and heat transfer in a power-law fluid past a vertical stretching sheet in the presence of a non-uniform heat source.Afzal et al.[3] studied momentum and transport on a continuous flat surface moving in a parallel stream. Anderson et al [4] treated film flow of power-law fluids. Chappidi and Gunnerson [5] treated analysis of heat and momentum transport along a moving surface. Anuar and Norfifah [6] studied power-law fluid flow on a moving wall. However, many practical situations demand for fluids that are non-Newtonian in nature such as molten plastics, polymeric liquid food stuffs and slurries.

In this paper we present a numerical analysis of the flow behavior when $n=1$ (Newtonian fluids) using finite difference method.

\section{Mathematical Formulation}

Consider a steady, two- dimensional laminar flow of a power-law fluid passing a moving flat plate with constant velocity $\mathrm{U}_{\mathrm{w}}$, in the same or opposite direction to the free stream

$U_{\infty}$.The $x$-axis extends parallel to the plate, while the $y$-axis extends upwards, normal to it. The boundary layer equations governing the flow in a power-law are [8],[2] and [9].

The steady equations are

$$
\begin{aligned}
& \frac{\partial u}{\partial x}+\frac{\partial v}{\partial y}=0 \\
& u \frac{\partial u}{\partial x}+v \frac{\partial u}{\partial y}=\frac{1}{\rho} \frac{\partial \tau x y}{\partial y}
\end{aligned}
$$

$$
\text { and }{ }^{v} \text { are velocity components along the }{ }^{x}{ }_{\text {and }}^{y} \text { axes, respectively }{ }^{\tau_{x y}} \text { is the shear stress, and } \rho \text { is }
$$

the fluid density. The boundary conditions are

$$
\begin{aligned}
& \begin{array}{l}
u=U_{w}, v=0 \\
y=0, u \rightarrow U_{\infty}
\end{array} \\
& \quad y \rightarrow \infty
\end{aligned}
$$

The stress tensor is defined as [7] and [4]

Where 


$$
\begin{aligned}
& \tau_{i j}=2 k\left|2 D_{k l} D_{k l}\right|\left(\frac{n-1 / 2)}{2} D_{i j}||\right. \\
& D_{i j}=\frac{1}{2}\left|\frac{\partial u_{i}}{\partial x_{j}}+\frac{\partial u_{j}}{\partial x_{i}}\right|
\end{aligned}
$$

Denotes the stretching tensor is called the coefficient and $\mathrm{n}$ is the power-law index. The index $\mathrm{n}$ is nondimensional, and the dimension of $k$ depends on the value of $n$.The two parameter rheological Eq.(2.4) is known as the Ostwald-de-Waele model or the power-law model.

The parameter $\mathrm{n}$ is an important index to subdivide fluids into pseudo plastic fluids $(\mathrm{n}<1)$, dilatants fluid $(n>1)$ and for $n=1$, the fluids is Newtonian. Therefore, the deviation of $n$ from unity indicates the degree of deviation from Newtonian behavior[9],Eq.(2.4) represents shear-thinning(n<1) and shear-thickening(n>1) fluids.

Using Eqs.(2.4)and (2.5),the shear stress appearing in Eq.(2.2) can be written as Now the momentum Eq.(2) becomes

$$
u \frac{\partial u}{\partial x}+v \frac{\partial u}{\partial y}=\frac{k}{\rho} \frac{\partial}{\partial y}\left(\left|\frac{\partial u}{\partial y}\right|^{n-1} \frac{\partial u}{\partial y}\right)
$$

The continuity equation (2.1) is satisfied by introducing a stream function $\psi_{\text {such that }}$

$$
u=\frac{\partial \psi}{\partial y}, v=-\frac{\partial \psi}{\partial x}
$$

The momentum equation can be transformed into the corresponding ordinary differential equation by introducing the following new variable [8]

$\eta=\left(\frac{\operatorname{Re}}{x / l}\right)^{\frac{1}{n+1}} \frac{y}{l}, \psi=l U_{\infty}\left(\frac{x / l}{\operatorname{Re}}\right)^{\frac{1}{n+1}} f(\eta)$,

$$
\begin{gathered}
\tau_{x y}=k\left|\frac{\partial u}{\partial y}\right|^{n-1} \quad \frac{\partial u}{\partial y} \quad \begin{array}{c}
\text { Where } \eta_{\text {is the similarity variable, }} f(\eta)_{\text {is the dimensionless stream function,L is }} \\
\text { the characteristic length and Re is the generalized Reynolds number defined as }
\end{array} \\
\operatorname{Re}=\frac{\rho U_{\infty}^{2-n} L^{n}}{k}
\end{gathered}
$$

differentiating Eqs.(2.8)\&(2.9) to get

$u=U_{\infty} f^{\prime}$

$\frac{\partial u}{\partial y}=U_{\infty} f^{\prime \prime}$

$\left|\frac{\partial u}{\partial y}\right|^{n-1}=U_{\infty}^{n-1}\left|f^{\prime \prime}\right|^{n-1}$

$\left|\frac{\partial u}{\partial y}\right|^{n-1} \frac{\partial u}{\partial y}=U_{\infty}^{n}\left|f^{\prime \prime}\right|^{n-1} f^{\prime \prime}$ 
Substitute Eqs.(2.3)\&(2.11)-(2.13) into (2.7) to get

$\left(\left|\frac{d^{2} f}{d \eta^{2}}\right|^{n-1} f^{\prime \prime}\right)^{\prime}+\frac{1}{n+1} f f^{\prime \prime}=0$

Where prime denote differentiation with respect to $\eta$

The transformed boundary conditions are

$$
f(0)=0, f^{\prime}(0)=\varepsilon, f^{\prime}(\eta) \rightarrow 1, \text { as } \eta \rightarrow \infty, f^{\prime}(\infty)=1
$$

Where $\varepsilon=\frac{U_{w}}{U}$ is the velocity ratio parameter.

\section{Method of Solution}

In order to solve the problem and keep it tractable, the set of non-linear ordinary differential equations (2.14) subject to boundary conditions (2.15) have been solved numerically using finite difference method.

We need some other conditions

$$
f^{\prime \prime}=f^{\prime \prime \prime}=-1
$$

Integrating Eq.(2.11) together with the boundary conditions (3.1) to get

$\left(\left|\frac{d^{2} f}{d \eta^{2}}\right|^{n-1} f^{\prime \prime}\right)+\frac{1}{n+1} f f^{\prime}=-1$

Let $n=1_{\text {in Eq.(3.2) to get }}$

$\left(f^{\prime \prime}\right)+\frac{1}{2} f f^{\prime}+1=0$

IV. Result

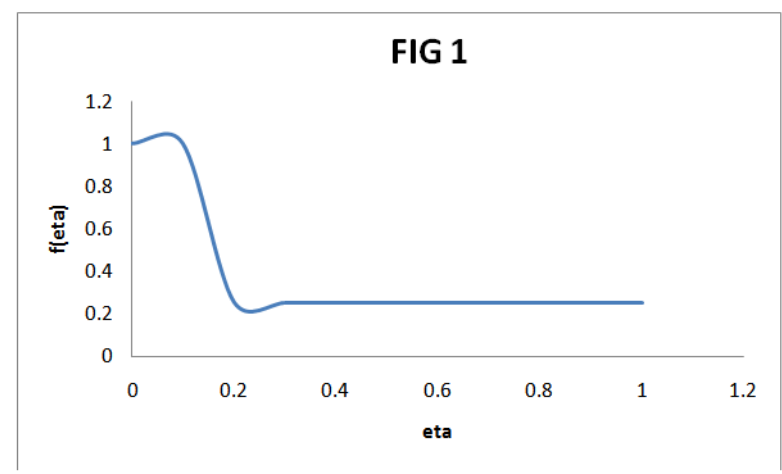

Fig4.1: Graph of the velocity function $f$ against the similarity variable $\eta$

when $n=1$.

\section{Conclusion}

We obtained a suitable expression for the steady boundary- layer flow of a power-law fluid on a moving wall. The power-law viscosity index $n$ subdivide the fluid into Newtonian fluid, the upper branch solutions are physically stable, while the lower branch solutions are not. We plot the graph of velocity function $f$ against the similarity variable $\eta$. It is seen from the graph that parameter $n$ affects the flow characteristics. 


\section{Reference}

[1] Abdelhafez,T.A;1985.Skin friction and heat transfer on a continuous flat surface moving in a parallel stream.Int.J.Heat Mass Transfer 28,pp.1234-1237.

[2] Abel,M.S;P.G.Siddheshwar and N.Mahesha,2009.Effects of thermal buoyancy and variable thermal conductivity on the MHD flow and heat transfer in a power-law fluid past a vertical stretching sheet in the presence of a non-uniform heat source.Int.J.Non-Linear Mech.44,pp.1-12.

[3] Afzal,N;A.Badaruddin and A.A.Elgarvi,1993.Momentum and transport on a continuous flat surface moving in a parallel stream.Int.J.Heat Mass Transfer 36,pp.3399-3403.

[4] Andersson,H.I.and F.Irgens,1990.Film flow of power-law fluids.In:Encyclopedia of Fluid Mechanics,Vol.9,Polymer Flow Engineering(N.P.Cheremissinoff,ed.).Gulf Publishing, Houston.

[5] Chappidi,P.R.and F.S.Gunnersson,1989.Analysis of heat and momentum transport along a moving surface.Int.J.Heat Mass Transfer 32,pp.1383-1386.

[6] Anuar Ishak and Norfifah Backok.2009.Power-law Fluid Flow on a Moving Wall. European Journal Scientific Research,Vol.34 No 1,pp.55-60.

[7] Wilkinson W.L,1960. Non-Newtonian Fluids. Pergamon Press, London.

[8] Wang,T.Y.,1994.Similarity solution of laminar mixed convection heat transfer from a horizontal plate to power-law fluid.Mingchi Inst.Tech.J.26,pp. 25-32.

[9] Mahapatra ,T.R.,S.K.Nandy and A.S.Gupta,2009.Magnetohydrodynamics stagnation point flow of a power-law fluid towards a stretching surface. Int. J. Non-Linear Mech.44, pp.124-129. 